\title{
Considerations in Mineral Processing of Scale-up from Bench to Pilot Plant to Full Production
}

\author{
Maria Sinche Gonzalez ${ }^{1}$, Saija Luukkanen ${ }^{2}$, Marko Paavola ${ }^{3}$ \\ ${ }^{1,2}$ Oulu Mining School/University of Oulu \\ P.O. Box 3000, Oulu, Finland \\ maria.sinchegonzalez@oulu.fi; saija.luukkanen@oulu.fi \\ ${ }^{3}$ Control Engineering/University of Oulu \\ marko.paavola@oulu.fi
}

\section{Extended Abstract}

Pilot plants are used to reduce the risk of scaling laboratory results to full scale production. Material handling, solidliquid separations, recycling streams (mediums), waste handling, recycled water as well as process control can be addressed in the pilot plant before the demonstration and commercial plants are built. These issues are difficult to investigate during bench scale tests.

For example, in flotation, laboratory scale tests have to be performed as completely and carefully as possible not only to optimize the metallurgical performance but also to tackle hydrodynamic issues and changes in the surface of mineral particles with the interaction of reagents, water constituents and air bubbles that would affect collection efficiency. Those are important considerations as chemistry and process will vary from plant to plant [1].

Additionally, pilot plant testing enables evaluating the performance of the process based on the preceding laboratory scale testing and against mechanical and operating difficulties that may arise; aiming at optimized continuous mode process operations before a full scale plant development. Additionally, a full automatized pilot plant will allow adjusting operational conditions due to changes in mineral composition, liberation (PSD, association, texture, others), oversee any variation on the process [2] and predict with more accuracy the final product at full scale.

A continuous pilot plant also enables to study the most suitable measurements and process control systems. Depending on the availability and cost of a measurement either direct, indirect or even novel measurements can be experimented. As an example, a dynamic model of a copper flotation circuit has been developed in Seppälä et al. work [3]. The model can be applied e.g. for studying the complex flow arrangements and back-circulations that need to be taken into account in control design. An additional advantage of the pilot plant is that it might reduce cost and time of developing advanced process control (APC) system for a plant. As reported in [4] the development of an optimal-setting control for grinding circuit, although providing significant savings and fast payback time, took over 20 months and 2 million dollars.

The objectives of this paper are first to show the importance of mineralogical and R\&D data from laboratory batch flotation testing, demonstration at pilot plant scale and the interpretation and conversion of the data into practical design requirements and consideration of scalability factors. Secondly, to emphasize the need of reliable information in scaling up from bench to pilot and further to full scale by modelling and, finally, to present the key considerations of scale-up and results obtained in the work done with $\mathrm{Cu}-\mathrm{Zn}$ ore from First Quantum Mineral's Pyhäsalmi mine in a continuous and completely automatized pilot plant at Oulu Mining School at University of Oulu, Finland. A series of batch flotation test were used to evaluate the behaviour of main ore minerals (copper, zinc). Obtained data was used to create floatability component models that were further applied in simulation by HSC Chemistry 7.1 to give a forecast of grades and recoveries in full scale production. Both the plant and the automation system are designed modular and can therefore be applied for various types of ores and beneficiation process flowsheets. 


\section{References}

[1] J. Connolly, G. Dobby, "Benchmarking of flotation plants: a key element of flotatiion modelling," In Recent Advances in Mineral Processing Plant Design; SME: Colorado, pp. 211-219, 2009.

[2] B. Yarar, Z. Dogan, Mineral Processing Design. Martinus Nijhoff Publishers: Netherlands, 1987.

[3] P. Seppälä, A. Sorsa, M. Paavola, J. Ruuska, A. Remes, H. Kumar, P. Lamberg, K. Leiviskä, "Development and calibration of a dynamic flotation circuit model," Miner. Eng., vol. 96-97, pp. 168-176, 2016.

[4] P. Zhou, T. Chai, H. Wang, "Intelligent optimal setting control for grinding circuits of mineral processing," IEEE Trans. Autom. Sci. Eng., vol. 6, pp. 730-743, 2009. 